\title{
Saddlepoint Approximations to Tail Probabilities and Quantiles of Inhomogeneous Discounted Compound Poisson Processes with Periodic Intensity Functions
}

\author{
Riccardo Gatto
}

Received: 23 July 2010 / Revised: 8 February 2011 /

Accepted: 14 February 2011 / Published online: 3 March 2011

(C) Springer Science+Business Media, LLC 2011

\begin{abstract}
This article provides saddlepoint approximations to tail probabilities and quantiles of the insurer discounted total claim amount, where the individual claim amounts are independent with a linear combination of exponential distributions and the number of claims is given by an inhomogeneous Poisson process with a periodic intensity function. It extends some previous results by Gatto (Methodol Comput Appl Probab 12:533-551, 2010), which are given for tail probabilities only and for non-periodic intensities only. Both extensions proposed in this article are important in the actuarial practice, where phenomena generating claims are subject to seasonal variations and where the quantiles or the values-at-risk of the total claim amount are desired. Some numerical comparisons of the new methods with Monte Carlo simulation are shown. The methods proposed are numerically very accurate, computationally efficient and hence relevant for the actuarial practice.
\end{abstract}

Keywords Circular distribution • Cumulant generating function • Flat-topped distribution • Fourier series • Interest rate • Monte Carlo simulation • Total claim amount • Trigonometric polynomial • von Mises distribution • Wrapped $\alpha$-stable distribution

AMS 2000 Subject Classifications $60 \mathrm{G} 55 \cdot 41 \mathrm{~A} 60$

This research was conducted while the author was visiting associate professor at the Department of Statistics and Applied Probability, University of California, Santa Barbara.

R. Gatto $(\varangle)$

Department of Mathematics and Statistics, Institute of Mathematical Statistics and Actuarial Science, University of Bern, Alpeneggstrasse 22, 3012 Bern, Switzerland e-mail: gatto@stat.unibe.ch 


\section{Introduction}

The Poisson process is currently used in actuarial science to count the number of claims and the compound Poisson process is used to evaluate the total claim amount, arising from a portfolio of risks over a given period. Many real phenomena generating claims are time inhomogeneous, and are also subject to periodic variations. Claim occurrences are often affected by weather conditions, whose periodicity is the year. Weather factors are indeed influential in fire, automobile liability and health insurance, for example. In these situations, the Poisson process with periodic intensity function is a sensible choice. Besides the time inhomogeneity, it is often important in actuarial planing to consider the force of interest in the evaluation of the total claim amount.

This article provides accurate analytical approximations to tail probabilities and quantiles of the total claim amount based on inhomogeneous Poisson processes with periodic intensities and subject to a fixed force of interest. In the first part of the article we propose some general methods for constructing periodic intensities of Poisson processes. For each periodic intensity proposed, we derive the cumulant generating function (c.g.f.) of the discounted total claim amount, when the individual claim amounts are independent and their distribution is given by a linear combination of exponential distributions. We then propose accurate approximations to the distribution function and to the quantiles of the inhomogeneous and discounted total claim amounts proposed, by inverting their c.g.f. with the saddlepoint approximation of asymptotic analysis.

This article is a continuation of developments by Gatto (2010), which are limited to nonperiodic intensities and to approximations of tail probabilities only. Other important references concerning distributions of total claim amounts are cited in Section 1 of Gatto (2010). Two further references are the following. Albrecher and Asmussen (2006) consider the total claim amount under a Poisson process with a shot-noise intensity process, for which they computed the moment generating function (m.g.f.). Léveillé et al. (2010) compute m.g.f. of various total claim amounts under a constant force of interest and a renewal counting process, thus without time inhomogeneity.

In Section 2 we propose various types of periodic intensity functions, and for each of these we provide the c.g.f. of the associated discounted total claim amount. In Section 3 we summarize the saddlepoint approximation to tail probabilities of the total claim amount, and then we propose an approximation for the quantiles or the values-at-risk. In Section 4 we show the numerical performance of the methods proposed by comparisons with Monte Carlo simulation. In Section 5 we give some final remarks.

\section{Periodic Intensities and Cumulant Generating Functions}

Suppose $X_{1}, X_{2}, \ldots>0$ are independent and identically distributed (i.i.d.) individual claim amounts, $0<T_{1}<T_{2}<\ldots$ are the corresponding times of claim occurrences, which are supposed independent of the individual claim amounts and which arise from an inhomogeneous Poisson process on $\mathbb{R}_{+}$. Let us define the number of claims occurring in the time interval $[0, t]$, for some $t>0$, by $N_{t}=\max \left\{k \geq 0 \mid T_{k} \leq t\right\}$, where 
$T_{0} \stackrel{\text { def }}{=} 0$ for convenience. We are interested on the total claim amount subject to the constant force of interest $\pm r \in \mathbb{R}$, which can be written as the shot-noise process

$$
Z_{t}=\sum_{i=0}^{N_{t}} e^{r\left(t-T_{i}\right)} X_{i}
$$

with $X_{0} \stackrel{\text { def }}{=} 0$. Assuming $r>0, Z_{t}$ is the total claim amount generated during $[0, t]$ and under compounding to the future time $t$ by the constant force of interest $r$. Alternatively, assuming $r<0, Z_{t}$ is the total claim amount generated during $[0, t]$ under discounting to the present time 0 by the constant force of interest $-r$. Many Poisson processes of insurance take place in a periodic environment and it is therefore sensible to consider periodic intensities, as defined below. Denote by $\tau \in(0, \infty)$ the period length (for example the year). A $\tau$-periodic Poisson process is characterized by the expectation function $\Lambda: \mathbb{R}_{+} \rightarrow \mathbb{R}_{+}, s \mapsto \mathrm{E}\left[N_{s}\right]$, satisfying $\Lambda(0)=0$ and

$$
\Lambda(s+\tau)-\Lambda(s)=\Lambda(\tau), \quad \forall s \geq 0 .
$$

Because $\Lambda$ is absolutely continuous with respect to (w.r.t.) the Lebesgue measure, there exists a density function $\lambda$, called intensity function of the process, satisfying $\Lambda\left(s_{2}\right)-\Lambda\left(s_{1}\right)=\int_{s_{1}}^{s_{2}} \lambda(s) d s$, for all $0 \leq s_{1}<s_{2}<\infty$. It follows from Eq. 1 that $\lambda$ is $\tau$ periodic. By defining the winding number

$$
w=\frac{t-t \bmod \tau}{\tau} \in \mathbb{N}
$$

we have

$$
\Lambda(t)=w \Lambda(\tau)+\Lambda(t \bmod \tau),
$$

and $\mathrm{P}\left[N_{j \tau+s}-N_{j \tau}=k\right]=\mathrm{P}\left[N_{s}=k\right]=e^{-\Lambda(s)} \Lambda^{k}(s) / k !, \forall s \geq 0$, and $j, k=0,1, \ldots$

We now give two general ways of constructing periodic intensities. The first one considers the nonnegative $\tau$-periodic finite Fourier series, or trigonometric polynomial, given by

$$
\lambda(s)=\alpha_{0}+\sum_{k=1}^{m}\left[\alpha_{k} \cos \left(\frac{2 \pi}{\tau} k s\right)+\beta_{k} \sin \left(\frac{2 \pi}{\tau} k s\right)\right],
$$

where $\alpha_{0}, \alpha_{1}, \ldots, \alpha_{m}, \beta_{1}, \ldots, \beta_{m} \in \mathbb{R}$ are selected so that $\lambda(s) \geq 0, \forall s \in[0, \tau)$, see Theorem 2.1 below. The intensity can be represented by a waveform with fundamental frequency $1 / \tau$ and harmonics, i.e. multiples of the fundamental frequency, with various amplitudes and various phases. If only a few harmonics are present, then the intensity can be represented by the trigonometric polynomial with fundamental frequency $1 / \tau$ (Eq. 4). From the theoretical point of view, this choice is motivated by the fact that the space of trigonometric polynomials is dense in the space of continuous periodic functions on $\mathbb{R}$; refer e.g. to Theorem 4.25 at p. 91 of Rudin (1987). Integrating Eq. 4 with the boundary condition $\Lambda(0)=0$ gives

$$
\Lambda(t)=\alpha_{0} t+\frac{\tau}{2 \pi} \sum_{k=1}^{m} \frac{1}{k}\left\{\alpha_{k} \sin \left(\frac{2 \pi}{\tau} k t\right)+\beta_{k}\left[1-\cos \left(\frac{2 \pi}{\tau} k t\right)\right]\right\},
$$


which is no longer a trigonometric polynomial unless $\alpha_{0}=0$. (But if $\alpha_{0}=0$ then $\lambda$ is not a nonnegative trigonometric polynomial.) The following theorem from Fejér (1915) gives necessary and sufficient conditions for the nonnegativity of a trigonometric polynomial. It is an equivalent form of the Fejér-Riesz representation; see Dimitrov (2002) for a review on nonnegative trigonometric polynomials.

Theorem 2.1 Nonnegativity of trigonometric polynomials (Fejér 1915).

The $\tau$-periodic trigonometric polynomial $\lambda(s)$ given in Eq. 4 is nonnegative for every $s \in[0, \tau)$ if and only if there exist $c_{0}, \ldots, c_{m} \in \mathbb{C}$ such that

$$
\alpha_{0}=\sum_{k=0}^{m}\left|c_{k}\right|^{2}, \alpha_{k}-i \beta_{k}=2 \sum_{j=0}^{m-k} c_{j+k} \bar{c}_{j}, \text { for } k=1, \ldots, m .
$$

Consider $m=2$ and denote $c_{k}=u_{k}+i v_{k}$, for $k=0,1,2$. Then the explicit forms of the coefficients are

$$
\begin{gathered}
\alpha_{0}=u_{0}^{2}+u_{1}^{2}+u_{2}^{2}+v_{0}^{2}+v_{1}^{2}+v_{2}^{2}, \\
\alpha_{1}=2\left(u_{0} u_{1}+u_{1} u_{2}+v_{0} v_{1}+v_{1} v_{2}\right), \beta_{1}=2\left(u_{1} v_{0}-u_{0} v_{1}-u_{1} v_{2}+u_{2} v_{1}\right), \\
\alpha_{2}=2\left(u_{0} u_{2}+v_{0} v_{2}\right) \text { and } \beta_{2}=2\left(v_{0} u_{2}-u_{0} v_{2}\right) .
\end{gathered}
$$

Although trigonometric polynomials represent the most flexible class of periodic intensities, the selection of the Fourier coefficients under the constraints of Theorem 2.1 is not always practical. Hence it is important to have an alternative source of periodic intensities. Probability "circular" densities provide a broad source of periodic intensities. The circular distribution function of a random angle $\theta$, measured in arc-length over the circle of circumference $\tau$, is defined by $F(x)=\mathrm{P}[0<\theta \leq x]$, if $x \in[0, \tau)$, and extended to $x \in \mathbb{R}$ by $F(x+\tau)-F(x)=1$. Thus any arc of length $\tau$ has probability 1 . This extension from $[0, \tau)$ to $\mathbb{R}$ is exploited for the construction of the expectation function (Eq. 10) below. If $\tau=2 \pi$, then we have the unit circle and $\theta$ is measured in radians. Thus $F(0)=0, F(\tau)=1$ and for $x_{1} \leq x_{2} \leq x_{1}+\tau$, $\mathrm{P}\left[x_{1}<\theta \leq x_{2}\right]=F\left(x_{2}\right)-F\left(x_{1}\right)=\int_{x_{1}}^{x_{2}} f(x) d x$, where the last equality holds if $F$ is absolutely continuous with density $f$ w.r.t. the Lebesgue measure on the circle of circumference $\tau$. Taking an affine transformation of any circular density $f$ on the circle of circumference $\tau$ gives the $\tau$-periodic intensity

$$
\lambda(s)=a_{0}+a_{1} f(s), \quad \forall s \geq 0,
$$

where $a_{0}$ and $a_{1}$ are such that $\lambda$ is nonnegative. The expectation function $\Lambda$ is $\lambda$ integrated with $\Lambda(0)=0$, namely

$$
\Lambda(t)=a_{0} t+a_{1} \int_{0}^{t} f(x) d x=a_{0} t+a_{1} F(t)
$$

There are various ways of obtaining circular distributions: by stereographic projection of $\mathbb{R}$ onto the circle, by radial projection of $\mathbb{R}^{2}$ towards $(0,0)$, giving the so-called "offset" distributions, by wrapping, giving the so-called "wrapped" distributions, and 
by considering characterizing properties, like maximum of entropy. Let $G$ denote an absolutely continuous distribution function with density $g$ w.r.t. the Lebesgue measure on $\mathbb{R}$. Wrapping $g$ around the circle of circumference $\tau$ yields the wrapped density

$$
f(s)=\sum_{j=-\infty}^{\infty} g(s+j \tau), \forall s \in \mathbb{R} .
$$

A $\tau$-periodic intensity $\lambda$ over $\mathbb{R}_{+}$can be obtained with the above wrapped density $f$ and Eq. 9. By defining the wrapped distribution function

$$
F(s)=\sum_{j=-\infty}^{\infty}[G(s+j \tau)-G(j \tau)], \quad \forall s \in \mathbb{R},
$$

the expectation function of $\lambda$ at time $t$ is given by $F$ and Eq. 10. The periodicity equation (Eq. 3) is clearly satisfied, as $\Lambda(t)=a_{0}(w \tau+t \bmod \tau)+a_{1}[w+F(t \bmod \tau)]=$ $w \Lambda(\tau)+\Lambda(t \bmod \tau)$. Two general references about circular distributions are Mardia and Jupp (2000) and Jammalamadaka and SenGupta (2001), where many examples can be found.

We are interested on the distribution of the discounted total claim amount $Z_{t}$ with a periodic intensity of the two types described and with the linear combination of exponentials individual claim amount density

$$
f_{X}(x)=\sum_{j=1}^{n} \mu_{j} v_{j} e^{-v_{j} x}, \quad \forall x>0,
$$

where $v_{1}, \ldots, v_{n}>0$ and the coefficients $\mu_{1}, \ldots, \mu_{n} \in \mathbb{R}$ are such that $f_{X}$ is a probability density on $\mathbb{R}_{+}$. A necessary condition for this is $\sum_{j=1}^{n} \mu_{j}=1$, whereas $\mu_{1}, \ldots, \mu_{n}>0$ is not necessary. We can obtain the form of the c.g.f. with the following lemma from Gatto (2010).

Lemma 2.1 Cumulant generating function of the discounted total claim amount (Gatto 2010).

Assume the m.g.f. of $X_{1}, M_{X}(v)=\mathrm{E}\left[e^{v X_{1}}\right]$, exists for all $v \in(-\infty, c)$, where $0 \leq c \leq$ $\infty$. Assume further that $\lambda(s)$ is continuous and bounded $\forall s \in[0, \tau)$. Then, the c.g.f. of $Z_{t}$ is given by

$$
K_{Z_{t}}(v)=\int_{0}^{t} M_{X}\left(v e^{r(t-y)}\right) \lambda(y) d y-\Lambda(t),
$$

$\forall t>0$ and $v<\gamma_{t} \stackrel{\text { def }}{=} c \min \left\{1, e^{-r t}\right\}$

Example 2.1 below gives an expansion of the c.g.f of the total claim amount, in the case where the intensity is represented by a trigonometric polynomial and under the linear combination of exponentials individual claim amount density (Eq. 11). The trigonometric polynomial intensity can be seen as a periodic version of the polynomial intensity given in Example 2.5 of Gatto (2010). Note the following errata in (14) of Gatto (2010): $n^{j+1}$ must be replaced $(n r)^{j+1}$ and $\Gamma(j+1, n t)$ by $\Gamma(j+1, n r t)$. 
Example 2.1 Trigonometric polynomial intensity and linear combination of exponentials claim amount.

Consider the trigonometric polynomial intensity function (Eq. 4), with coefficients satisfying the conditions of Theorem 2.1, and the linear combination of exponentials claim amount density (Eq. 11). Then we have

$$
K_{Z_{t}}(v)=\sum_{j=1}^{n} \mu_{j}\left(\frac{\alpha_{0}}{r} \log \frac{v_{j}-v}{v_{j} e^{-r t}-v}+\sum_{k=1}^{m} \alpha_{k} C_{k}\left(v, v_{j}\right)+\beta_{k} S_{k}\left(v, v_{j}\right)\right)-\Lambda(t),
$$

where $\Lambda(t)$ is given by Eq. 5 ,

$$
\begin{aligned}
C_{k}(v, v)= & \frac{\sin \left(\frac{2 \pi}{\tau} k t\right)}{\frac{2 \pi}{\tau} k} \\
& +\sum_{j=1}^{\infty} \frac{\left(\frac{v}{v}\right)^{j}}{(j r)^{2}+\left(\frac{2 \pi}{\tau} k\right)^{2}}\left\{\frac{2 \pi}{\tau} k \sin \left(\frac{2 \pi}{\tau} k t\right)+j r\left[e^{j r t}-\cos \left(\frac{2 \pi}{\tau} k t\right)\right]\right\}
\end{aligned}
$$

and

$$
\begin{aligned}
S_{k}(v, v)= & \frac{1-\cos \left(\frac{2 \pi}{\tau} k t\right)}{\frac{2 \pi}{\tau} k}+\sum_{j=1}^{\infty} \frac{\left(\frac{v}{v}\right)^{j}}{(j r)^{2}+\left(\frac{2 \pi}{\tau} k\right)^{2}} \\
& \times\left\{-j r \sin \left(\frac{2 \pi}{\tau} k t\right)+\frac{2 \pi}{\tau} k\left[e^{j r t}-\cos \left(\frac{2 \pi}{\tau} k t\right)\right]\right\},
\end{aligned}
$$

$\forall|v|<\gamma_{t} \stackrel{\text { def }}{=} \min \left\{v_{1}, \ldots, v_{n}\right\} \min \left\{1, e^{-r t}\right\}, r \neq 0$ and $k=1, \ldots, m$.

Proof We mainly need to compute three types of integrals. The first one is

$$
\int_{0}^{t} \frac{1}{1-\frac{v}{v} e^{r(t-y)}} d y=\frac{1}{r} \log \frac{v-v}{v e^{-r t}-v}
$$

$\forall v>0, v<v \min \left\{1, e^{-r t}\right\}$ and $r \neq 0$, with details given in Example 2.1 of Gatto (2010). The second type of integral is

$$
\int_{0}^{t} \frac{1}{1-\frac{v}{v} e^{r(t-y)}} \cos \left(\frac{2 \pi}{\tau} k y\right) d y=\sum_{j=0}^{\infty}\left(\frac{v}{v} e^{r t}\right)^{j} \int_{0}^{t} e^{-j r y} \cos \left(\frac{2 \pi}{\tau} k y\right) d y,
$$

$\forall v>0,|v|<v \min \left\{1, e^{-r t}\right\}$ and $k=1, \ldots, m$, where the last expression above can be simplified to $C_{k}(v, v)$ in Eq. 13 by noting that

$$
\int e^{a x} \cos b x d x=\frac{1}{2} \int e^{a x}\left(e^{i b x}+e^{-i b x}\right) d x=\frac{e^{a x}}{a^{2}+b^{2}}(a \cos b x+b \sin b x) .
$$

The third type of integral is

$$
\int_{0}^{t} \frac{1}{1-\frac{v}{v} e^{r(t-y)}} \sin \left(\frac{2 \pi}{\tau} k y\right) d y=\sum_{j=0}^{\infty}\left(\frac{v}{v} e^{r t}\right)^{j} \int_{0}^{t} e^{-j r y} \sin \left(\frac{2 \pi}{\tau} k y\right) d y,
$$


$\forall v>0,|v|<v \min \left\{1, e^{-r t}\right\}$ and $k=1, \ldots, m$, where the last expression above can be simplified to $S_{k}(v, v)$ in Eq. 14 by noting that

$$
\int e^{a x} \sin b x d x=\frac{1}{2 i} \int e^{a x}\left(e^{i b x}-e^{-i b x}\right) d x=\frac{e^{a x}}{a^{2}+b^{2}}(a \sin b x-b \cos b x) .
$$

The three integration formulae above together with Lemma 2.1 lead to the c.g.f. in Eq. 12.

Note that setting $\alpha_{k}=\beta_{k}=0$, for $k=1, \ldots, m$, into Eq. 12 gives (4) in Gatto (2010).

We now consider intensities based on circular densities as in Eq. 9 and expand them in Fourier series, in order to obtain expansions of the integral representation of the c.g.f of $Z_{t}$ of Lemma 2.1. Assume that the circular density $f$ belongs to $\mathcal{L}_{2}[0, \tau)$, the Hilbert space of square integrable functions on $[0, \tau)$ with inner product $\int_{0}^{\tau} f(x) g(x) d x$. Then we have the rescaled Fourier expansion

$$
\begin{aligned}
f(s) & =\frac{1}{\tau} \sum_{k=-\infty}^{\infty} \varphi_{k} \exp \left\{-i \frac{2 \pi}{\tau} k s\right\} \\
& =\frac{1}{\tau}\left\{1+2 \sum_{k=1}^{\infty}\left[\gamma_{k} \cos \left(\frac{2 \pi}{\tau} k s\right)+\delta_{k} \sin \left(\frac{2 \pi}{\tau} k s\right)\right]\right\},
\end{aligned}
$$

$\forall s \in \mathbb{R}$, where $\varphi_{k}=\gamma_{k}+i \delta_{k}, \forall k \in \mathbb{Z}$. Clearly,

$$
\gamma_{k}=\int_{0}^{\tau} \cos \left(\frac{2 \pi}{\tau} k s\right) f(s) d s, \delta_{k}=\int_{0}^{\tau} \sin \left(\frac{2 \pi}{\tau} k s\right) f(s) d s
$$

and

$$
\varphi_{k}=\int_{0}^{\tau} \exp \left\{i \frac{2 \pi}{\tau} k s\right\} f(s) d s, \quad \forall k \in \mathbb{Z} .
$$

The equality in Eq. 16 is meant in the $\mathcal{L}_{2}$-sense and the series in the right-handside of Eq. 16 converges to $f$ uniformly, if $f$ is $\mathcal{C}_{1}$. Also, $\lim _{|k| \rightarrow \infty} \varphi_{k}=0$, from the Riemann-Lebesgue lemma. If $f$ is obtained by wrapping the density of the random variable $L \in \mathbb{R}$ around the circle of circumference $\tau$, then the $k$ th Fourier coefficient or trigonometric moment is obtained from

$$
\varphi_{k}=\varphi_{L}(2 \pi / \tau k),
$$

where $\varphi_{L}(v)=\mathrm{E}[\exp \{i v L\}], \forall v \in \mathbb{R}$, namely the $k$ th Fourier coefficient is the characteristic function (c.f.) of $L$ evaluated at the fractional values $2 \pi / \tau k, \forall k \in \mathbb{Z}$. This follows from

$$
\begin{aligned}
\varphi_{k} & =\int_{0}^{\tau} \exp \left\{i \frac{2 \pi}{\tau} k s\right\} \sum_{j=-\infty}^{\infty} g(s+j \tau) d s=\sum_{j=-\infty}^{\infty} \int_{0}^{\tau} \exp \left\{i \frac{2 \pi}{\tau} k(s+j \tau)\right\} g(s+j \tau) d s \\
& =\sum_{j=-\infty}^{\infty} \int_{j \tau}^{(j+1) \tau} \exp \left\{i \frac{2 \pi}{\tau} k s\right\} g(s) d s=\int_{-\infty}^{\infty} \exp \left\{i \frac{2 \pi}{\tau} k s\right\} g(s) d s,
\end{aligned}
$$


where the change of order of summation and integration can be justified by the Monotone convergence theorem. Result 2.1 below gives an expansion to the c.g.f. of the discounted total claim amount.

Result 2.1 Expansion of the cumulant generating function for a linear combination of exponentials claim amount.

Let $f$ be a $\mathcal{C}_{1}$ circular density in $\mathcal{L}_{2}[0, \tau)$ with Fourier series (Eq. 16) and consider the intensity $\lambda(s)=a_{0}+a_{1} f(s)$, with $a_{0}, a_{1}$ chosen such that $\lambda(s) \geq 0, \forall s \in[0, \tau)$. Consider also the linear combination of exponentials claim amount density given by Eq. 11. Then the c.g.f. of $Z_{t}$ can be expanded as

$$
K_{Z_{t}}(v)=\sum_{j=1}^{n} \mu_{j}\left(\frac{\alpha_{0}}{r} \log \frac{v_{j}-v}{v_{j} e^{-r t}-v}+\sum_{k=1}^{\infty} \alpha_{k} C_{k}\left(v, v_{j}\right)+\beta_{k} S_{k}\left(v, v_{j}\right)\right)-\Lambda(t),
$$

$\forall|v|<\gamma_{t} \stackrel{\text { def }}{=} \min \left\{v_{1}, \ldots, v_{n}\right\} \min \left\{1, e^{-r t}\right\} \quad$ and $r \neq 0$, where $\alpha_{0}=a_{0}+a_{1} / \tau, \quad \alpha_{k}=$ $2 a_{1} / \tau \gamma_{k}, \beta_{k}=2 a_{1} / \tau \delta_{k}, C_{k}(v, v)$ and $S_{k}(v, v)$ are given by Eqs. 13 and 14, respectively, now for $k=1,2, \ldots$, and $\Lambda(t)$ is given by Eq. 5 with $m=\infty$.

Proof The first part of this proof follows the same steps of the proof of the expansion of the c.g.f in Eq. 12 of Example 2.1, under the trigonometric polynomial intensity. We then let the degree $m$ of the Fourier polynomial go to $\infty$ and justify the change of order of summation and integration by the uniform convergence of the Fourier series of $f$, which is implied by the assumption that $f$ is $\mathcal{C}_{1}$. Next, integrating term by term the Fourier series of a piecewise continuous periodic function yields a series converging uniformly to the integral of the function; see e.g. Theorem 2.27 at p. 77 of Pinkus and Zafrany (1997). Hence term by term integration of the Fourier series of the $\tau$-periodic and $\mathcal{C}_{1}$ intensity $\lambda$ gives the series obtained by the right side of Eq. 5 with $m=\infty$, which converges uniformly to $\Lambda(t)=\int_{0}^{t} \lambda(s) d s$.

The remaining part of this section proposes periodic intensities constructed from some circular distributions, together with expansions of the c.g.f. of their discounted total claim amount $Z_{t}$, under the linear combination of exponentials individual claim amount distribution. All circular distributions given here are unimodal and a short guidance for choosing them is the following. An important result is given by Example 2.2. It gives a wide class of symmetric and asymmetric densities, which can have either sharp or Gaussian shaped modes and various types of decay from the mode. They are based on the wrapped $\alpha$-stable $(\mathrm{W} \alpha \mathrm{S})$ distribution. The shapes of these densities are governed by three parameters. Example 2.3 is based on the von Mises distribution, which has two parameters. The densities are symmetric with Gaussian shaped modes. From the theoretical point of view, the von Mises distribution is a maximum entropy distribution. Example 2.4 is based on a symmetric circular distribution which can have a wide and flat part at the level of the mode. This particular shape of the density can neither be obtained by the $\mathrm{W} \alpha \mathrm{S}$ nor by the von Mises densities. This distribution has three parameters and it is due to Batschelet (1981). Example 2.5 is based on a particular asymmetric distribution which allows for inflexion points. It has three parameters and it is also due to Batschelet (1981). Finally, Example 2.6 is based on the wrapped exponential density. It has one parameter, it decreases steadily over each entire period and jumps upwards at the beginning of the next period. 
An important class of unimodal circular distributions is the $\mathrm{W} \alpha \mathrm{S}$; refer to Gatto and Jammalamadaka (2003). It derives from wrapping $\alpha$-stable distributions from $\mathbb{R}$ onto the unit circle. We denote an $\alpha$-stable random variable by $S_{\alpha}(\sigma, \beta, \mu) \in$ $\mathbb{R}$, where $\alpha \in(0,2], \beta \in[-1,1], \sigma \in \mathbb{R}_{+}$and $\mu \in \mathbb{R}$ are the parameters of stability (shape), skewness, scale and shift, respectively. When $\beta=0, S_{\alpha}(\sigma, 0, \mu)$ is symmetric about $\mu$. Two well-known $\alpha$-stable random variables are the normal with mean $\mu$ and variance $2 \sigma^{2}$, denoted as $S_{2}(\sigma, 0, \mu)$ or as $\mathcal{N}\left(\mu, 2 \sigma^{2}\right)$, and the Cauchy $S_{1}(\sigma, 0, \mu)$, with density function $2 \sigma /\left(\pi\left[(x-\mu)^{2}+4 \sigma^{2}\right]\right)$. For a complete presentation, see e.g. Samorodnitsky and Taqqu (1994). WS $\alpha \mathrm{S}$ densities are unimodal with various shapes: symmetric or asymmetric, with sharp or smooth modes, and with heavy or light tails. The graphs of some densities are shown in Figure 1 of Gatto and Jammalamadaka (2003). Example 2.2 below gives the trigonometric moments of $\mathrm{W} \alpha \mathrm{S}$ distributions, which allow to compute the expansion of the c.g.f. of the discounted total claim amount.

Example 2.2 Wrapped $\alpha$-stable intensity and linear combination of exponentials claim amount.

Consider the intensity (Eq. 9), where $f$ is the wrapped density of $S_{\alpha}(\sigma, \beta, \mu)$ around the circle of circumference $\tau$, with $\alpha \neq 1$. Consider also the linear combination of exponentials claim amount density given by Eq. 11. Then the c.g.f. of $Z_{t}$ can be expanded as Eq. 18 in Result 2.1, where for $k=1,2, \ldots$,

$$
\gamma_{k}=\exp \left\{-\left(\sigma \frac{2 \pi}{\tau} k\right)^{\alpha}\right\} \cos \left\{\left(\sigma \frac{2 \pi}{\tau} k\right)^{\alpha} \beta \tan \frac{\alpha \pi}{2}+\frac{2 \pi}{\tau} \mu k\right\}
$$

and

$$
\delta_{k}=\exp \left\{-\left(\sigma \frac{2 \pi}{\tau} k\right)^{\alpha}\right\} \sin \left\{\left(\sigma \frac{2 \pi}{\tau} k\right)^{\alpha} \beta \tan \frac{\alpha \pi}{2}+\frac{2 \pi}{\tau} \mu k\right\} .
$$

Also, the $\mathrm{W} \alpha \mathrm{S}$ density has the series representation

$$
\begin{aligned}
f(s)= & \frac{1}{\tau}\left\{1+2 \sum_{k=1}^{\infty} \exp \left\{-\left(\sigma \frac{2 \pi}{\tau} k\right)^{\alpha}\right\} \cos \left(\frac{2 \pi}{\tau} k(s-\mu)-\left(\sigma \frac{2 \pi}{\tau} k\right)^{\alpha} \beta \tan \frac{\alpha \pi}{2}\right)\right\}, \\
& \forall s \in \mathbb{R} .
\end{aligned}
$$

In the above expressions, $\mu$ is conveniently redefined as $\mu \stackrel{\text { def }}{=} \mu \bmod \tau$.

Proof $\mathrm{W} \alpha \mathrm{S}$ densities over the circle of circumference $\tau$ can be constructed via Eq. 16 by using the c.f. of $L \sim S_{\alpha}(\sigma, \beta, \mu)$, given by

$$
\varphi_{L}(v)=\left\{\begin{array}{l}
\exp \left\{-\sigma^{\alpha}|v|^{\alpha}\left(1-i \beta \operatorname{sgn} v \tan \frac{\alpha \pi}{2}\right)+i \mu v\right\}, \text { if } \alpha \in(0,1) \cup(1,2], \\
\exp \left\{-\sigma|v|\left(1+i \frac{2}{\pi} \beta \operatorname{sgn} v \log |v|+i \mu v\right)\right\}, \text { if } \alpha=1,
\end{array} \forall v \in \mathbb{R} .\right.
$$


Formula 17, which states that the trigonometric moments of a wrapped distribution correspond to the original c.f. at integer multiples of $2 \pi / \tau$, gives us the coefficients in Eqs. 19 and 20. We can then apply Result 2.1 with these coefficients. The coefficients in Eqs. 19 and 20 give also the wrapped density (Eq. 21) and so the intensity (Eq. 9).

From the above c.f. of $L$, we see that the case $\alpha=1$ can be solved in a similar way. Note also that when $\alpha=1 / 2$ and 2, and when $\alpha=1$ together with $\beta=0$, closed form expressions of the linear $\alpha$-stable densities are available. An alternative expansion to the c.g.f. of $Z_{t}$, which avoids the Fourier expansion, can be found for the case $\alpha=2$, namely for the wrappend normal density, by using the formula of the normal density.

In Example 2.3 we consider the von Mises density over the circle of circumference $\tau$

$$
f(s)=\frac{1}{\tau I_{0}(\kappa)} \exp \left\{\kappa \cos \left(\frac{2 \pi}{\tau}(s-\mu)\right)\right\}
$$

$\forall s \in \mathbb{R}, \mu \in[0, \tau), \kappa>0$, where $I_{k}(z)=(2 \pi)^{-1} \int_{0}^{2 \pi} \cos k \omega \exp \{z \cos \omega\} d \omega, z \in \mathbb{C}$, is the modified Bessel function of the first kind of integer order $k$; see 9.6 .19 at p. 376 of Abramowitz and Stegun (1972). The von Mises distribution has an important information theoretic property: it maximizes the entropy within all circular densities with a given first trigonometric moment, i.e. with fixed mean direction and mean resultant length. The maximum entropy principle tells the importance of this distribution. It states that, subject to known constraints, the distribution which best represents the current state of knowledge is the one with maximal entropy.

Example 2.3 von Mises intensity and linear combination of exponentials claim amount.

Consider the intensity (Eq. 9), where $f$ is the von Mises density (Eq. 22). Consider also the linear combination of exponentials claim amount density given by Eq. 11. Then the c.g.f. of $Z_{t}$ can be expanded as Eq. 18 in Result 2.1, where for $k=1,2, \ldots$

$$
\gamma_{k}=\frac{I_{k}(\kappa)}{I_{0}(\kappa)} \cos \left(k \frac{2 \pi}{\tau} \mu\right) \text { and } \delta_{k}=\frac{I_{k}(\kappa)}{I_{0}(\kappa)} \sin \left(k \frac{2 \pi}{\tau} \mu\right) .
$$

Proof Denoting by $\theta \in[0, \tau)$ a random angle with the density (Eq. 22), then for any $k \in \mathbb{Z}$, we have

$$
\begin{aligned}
\varphi_{k} & =\mathrm{E}\left[\exp \left\{i k \frac{2 \pi}{\tau} \theta\right\}\right]=\mathrm{E}\left[\exp \left\{i k \frac{2 \pi}{\tau}(\theta-\mu)\right\}\right] \exp \left\{i k \frac{2 \pi}{\tau} \mu\right\} \\
& =\mathrm{E}\left[\cos \left(k \frac{2 \pi}{\tau}(\theta-\mu)\right)\right] \exp \left\{i k \frac{2 \pi}{\tau} \mu\right\}=\frac{I_{k}(\kappa)}{I_{0}(\kappa)} \exp \left\{i k \frac{2 \pi}{\tau} \mu\right\},
\end{aligned}
$$

which gives Eq. 23, for $k=1,2, \ldots$.

Note that the Fourier coefficients in Eq. 23 can be computed recursively with $I_{k+1}(z)=I_{k-1}(z)-2 k / z I_{k}(z), \forall z \neq 0$. Note also that the generalized von Mises 
distribution of order $k$ maximizes the entropy within all circular distributions with given first $k$ trigonometric moments, for $k=1,2, \ldots$, where the case $k=1$ corresponds to the von Mises distribution; see Gatto and Jammalamadaka (2007) and Gatto (2008). The case $k=2$ allows for bimodality and asymmetry.

Another useful unimodal circular density is flat and wide at the level of the mode and steep around the antimode, which is the mode plus half of the period. It is given in Section 15.7 of Batschelet (1981). On the circle of circumference $\tau$, this density is given by

$$
f(s)=\frac{1}{\tau\left\{1-\kappa J_{1}(v)\right\}}\left\{1+\kappa \cos \left(\frac{2 \pi}{\tau}(s-\mu)+v \sin \left[\frac{2 \pi}{\tau}(s-\mu)\right]\right)\right\},
$$

$\forall s \in \mathbb{R}, \mu \in[0, \tau), v \in(-1,1)$ and $\kappa \in[-1,1]$, where $J_{k}(z)=(2 \pi)^{-1} \int_{0}^{2 \pi} \cos (k \omega-$ $z \sin \omega) d \omega, z \in \mathbb{C}$, is the Bessel function of the first kind of integer order $k$; see e.g. 9.1.21 at p. 360 of Abramowitz and Stegun (1972). The function $\omega+v \sin \omega$ is strictly monotonic in $\omega$ iff $v \in(-1,1)$. Thus, taking $v \in(-1,1)$ guarantees that $f^{\prime}(s)=0$ has two distinct solutions, when $s \in[0, \tau)$, and therefore that $f$ is an unimodal circular density. The nonnegativity of $f$ is ensured by $\kappa \in[-1,1]$.

We define

$$
\mathrm{I}\{A\}=\left\{\begin{array}{l}
1, \text { if the statement } A \text { is true, } \\
0, \text { otherwise }
\end{array}\right.
$$

as the indicator function.

Example 2.4 Flat-topped intensity and linear combination exponentials claim amount.

Consider the intensity (Eq. 9), where $f$ is the flat-topped circular density (Eq. 24). Consider also the linear combination of exponentials claim amount density given by Eq. 11. Then the c.g.f. of $Z_{t}$ can be expanded as Eq. 18 in Result 2.1, where $\forall v \in(-1,0) \cup(0,1)$ and $k=1,2, \ldots$,

$$
\begin{aligned}
\gamma_{k}(0) & =\frac{\kappa}{1-\kappa J_{1}(v)}\left[(-1)^{k-1} \frac{k}{v} J_{k}(v)+J_{k-1}(v) \mid\{k \in\{2,4, \ldots\}\}\right], \\
\gamma_{k} & =\gamma_{k}(0) \cos \left(\frac{2 \pi}{\tau} k \mu\right) \text { and } \delta_{k}=\gamma_{k}(0) \sin \left(\frac{2 \pi}{\tau} k \mu\right) .
\end{aligned}
$$

Proof By applying elementary trigonometric identities for sum of angles we obtain

$$
\begin{aligned}
& \int_{0}^{2 \pi} \cos (\omega+v \sin \omega) \cos k \omega d \omega \\
& =\frac{1}{2}\left\{\int_{0}^{2 \pi} \cos (v \sin \omega) \cos (k+1) \omega d \omega+\int_{0}^{2 \pi} \cos (v \sin \omega) \cos (k-1) \omega d \omega\right. \\
& \left.\quad \quad-\int_{0}^{2 \pi} \sin (v \sin \omega) \sin (k+1) \omega d \omega+\int_{0}^{2 \pi} \sin (v \sin \omega) \sin (k-1) \omega d \omega\right\},
\end{aligned}
$$


for $k=1,2, \ldots$ Inside the large parentheses on the right side of Eq. 26 we have four integrals where: the first one is $2 \pi J_{k+1}(v)$, if $k=1,3, \ldots$, and 0 , if $k=2,4, \ldots$; the second one is $2 \pi J_{k-1}(v)$, if $k=1,3, \ldots$, and 0 , if $k=2,4, \ldots$; the third one is 0 , if $k=1,3, \ldots$, and $2 \pi J_{k+1}(v)$, if $k=2,4, \ldots$; and the fourth one is 0 , if $k=1,3, \ldots$, and $2 \pi J_{k-1}(v)$, if $k=2,4, \ldots$ Indeed, these integrals are alternative representations of the Bessel function of the first kind and they can be directly constructed from the Jacobi expansions 9.1.42 and 9.1.43 at p. 361 of Abramowitz and Stegun (1972). With this, the integral on the left side of Eq. 26 is equal to

$$
\begin{cases}\pi\left\{J_{k-1}(v)+J_{k+1}(v)\right\}, & \text { if } k=1,3, \ldots \\ \pi\left\{J_{k-1}(v)-J_{k+1}(v)\right\}, & \text { if } k=2,4, \ldots\end{cases}
$$

which is also equal to

$$
2 \pi\left[(-1)^{k-1} \frac{k}{v} J_{k}(v)+J_{k-1}(v) \mid\{k=2,4, \ldots\}\right],
$$

because of

$$
J_{k+1}(z)=\frac{2 k}{z} J_{k}(z)-J_{k-1}(z), \forall z \neq 0, k=1,2, \ldots
$$

Denoting $\gamma_{k}=\gamma_{k}(\mu)$ and $\delta_{k}=\delta_{k}(\mu)$, we find $\delta_{k}(0)=0$, from the symmetry of the centered density, and $\gamma_{k}(0)$ equal to $\kappa /\left[2 \pi\left\{1-\kappa J_{1}(v)\right\}\right]$ times Eq. 27, for $k=1,2, \ldots$ Thus $\gamma_{k}(\mu)=\gamma_{k}(0) \cos (2 \pi / \tau k \mu)$ and $\delta_{k}(\mu)=\gamma_{k}(0) \sin (2 \pi / \tau k \mu), \forall \mu \in[0, \tau)$ and $k=$ $1,2, \ldots$, which is Eq. 25.

Note that the Fourier coefficients in Eq. 25 can be computed recursively with Eq. 28.

Next, a $\tau$-periodic unimodal and skewed intensity can be constructed from another circular distribution suggested by Batschelet (1981) in Section 15.6. Over the circle of circumference $\tau$, the density is given by

$$
f(s)=\frac{1}{\tau}\left\{1+\kappa \sin \left(\frac{2 \pi}{\tau}(s-\mu)+v \sin \left[\frac{2 \pi}{\tau}(s-\mu)\right]\right)\right\},
$$

$\forall s \in \mathbb{R}, \mu \in[0, \tau), v \in(-1,1)$ and $\kappa \in[-1,1]$. As in the previous example, the unimodality and the nonnegativity of $f$ are ensured by these restrictions on the parameters.

Example 2.5 Skewed intensity and linear combination exponentials claim amount.

Consider the intensity (Eq. 9), where $f$ is the skewed circular density (Eq. 29). Consider also the linear combination of exponentials claim amount density given by Eq. 11. Then the c.g.f. of $Z_{t}$ can be expanded as Eq. 18 in Result 2.1, where $\forall v \in$ $(-1,0) \cup(0,1)$ and $k=1,2, \ldots$,

$$
\begin{aligned}
\delta_{k}(0) & =\kappa\left[(-1)^{k} \frac{k}{v} J_{k}(v)+J_{k-1}(v) \mid\{k \in\{1,3, \ldots\}\}\right], \\
\gamma_{k} & =-\delta_{k}(0) \sin \left(\frac{2 \pi}{\tau} k \mu\right) \text { and } \delta_{k}=\delta_{k}(0) \cos \left(\frac{2 \pi}{\tau} k \mu\right) .
\end{aligned}
$$


Proof By applying elementary trigonometric identities for sum of angles we obtain

$$
\begin{aligned}
& \int_{0}^{2 \pi} \sin (\omega+v \sin \omega) \sin k \omega d \omega \\
& =\frac{1}{2}\left\{\int_{0}^{2 \pi} \cos (v \sin \omega) \cos (k-1) \omega d \omega-\int_{0}^{2 \pi} \cos (v \sin \omega) \cos (k+1) \omega d \omega\right. \\
& \left.\quad+\int_{0}^{2 \pi} \sin (v \sin \omega) \sin (k+1) \omega d \omega+\int_{0}^{2 \pi} \sin (v \sin \omega) \sin (k-1) \omega d \omega\right\},
\end{aligned}
$$

for $k=1,2, \ldots$ Inside the large parentheses on the right side of Eq. 31 we have four integrals where: the first one is $2 \pi J_{k-1}(v)$, if $k=1,3, \ldots$, and 0 , if $k=2,4, \ldots$; the second one is $2 \pi J_{k+1}(\nu)$, if $k=1,3, \ldots$, and 0 , if $k=2,4, \ldots$; the third one is 0 , if $k=1,3, \ldots$, and $2 \pi J_{k+1}(v)$, if $k=2,4, \ldots$; and the fourth one is 0 , if $k=1,3, \ldots$, and $2 \pi J_{k-1}(v)$, if $k=2,4, \ldots$ So the integral on the left side of Eq. 31 is equal to

$$
\begin{cases}\pi\left\{J_{k-1}(v)-J_{k+1}(v)\right\}, & \text { if } k=1,3, \ldots, \\ \pi\left\{J_{k-1}(v)+J_{k+1}(v)\right\}, & \text { if } k=2,4, \ldots,\end{cases}
$$

which is equal to

$$
2 \pi\left[(-1)^{k} \frac{k}{v} J_{k}(v)+J_{k-1}(v) \mid\{k=1,3, \ldots\}\right],
$$

from Eq. 28. Denoting $\gamma_{k}=\gamma_{k}(0)$ and $\delta_{k}=\delta_{k}(0)$, we find $\gamma_{k}(0)=0$, from the antisymmetry of the centered density, and $\delta_{k}(0)$ equal to $\kappa /(2 \pi)$ times Eq. 32, for $k=1,2, \ldots$ Thus $\gamma_{k}(\mu)=-\delta_{k}(0) \sin (2 \pi / \tau k \mu)$ and $\delta_{k}(\mu)=\delta_{k}(0) \cos (2 \pi / \tau k \mu), \forall \mu \in$ $[0, \tau)$ and $k=1,2, \ldots$, which is Eq. 30 .

In Example 2.6 we consider the wrapped exponential intensity, which is useful when the claim occurrence increases suddenly at the beginning of each period and then decreases steadily within each period. Consider the exponential density $g(s)=$ $\theta e^{-\theta s}$, with $s, \theta>0$. The wrapped version of $g$ around the circle of circumference $\tau$ is obtained by

$$
h(s)=\sum_{j=0}^{\infty} \theta e^{-\theta(s+j \tau)}=\frac{\theta e^{-\theta s}}{1-e^{-\theta \tau}}, \forall s \in[0, \tau),
$$

followed by the $\tau$-periodic continuation

$$
f(s)=h(s \bmod \tau), \forall s \in \mathbb{R} .
$$

Example 2.6 Wrapped exponential intensity and exponential claim amounts.

Consider the intensity (Eq. 9) where $f$ is the wrapped exponential density (Eq. 33). Consider also the exponential claim amount density $f_{X}(x)=v e^{-v x}, \forall x, v>0$. Then we have

$$
\begin{aligned}
K_{Z_{t}}(v)= & \frac{a_{0}}{r} \log \frac{v-v}{v-v e^{r t}}+\frac{a_{1} \theta}{1-e^{-\theta \tau}} \sum_{k=1}^{\infty} \frac{\left(\frac{v}{v} e^{r t}\right)^{k}}{k r+\theta} \\
& \times\left(\frac{\left(1-e^{-k r w t}\right)\left(e^{-\theta \tau}-e^{k r \tau}\right)}{1-e^{k r \tau}}+e^{-k r w \tau}-e^{-\theta(t-w \tau)-k r t}\right),
\end{aligned}
$$


$\forall|v|<\gamma \stackrel{\text { def }}{=} v \min \left\{1, e^{-r t}\right\}$ and $r \neq 0$, where $w$ is the winding number defined in Eq. 2.

Proof Assume $w \geq 1$, then

$$
\begin{aligned}
A(v, v) & \stackrel{\operatorname{def}}{=} \int_{0}^{t} \frac{1}{1-v^{-1} v e^{r(t-y)}} e^{-\theta(y \bmod \tau)} d y \\
= & \sum_{j=1}^{w}\left\{\int_{(j-1) \tau}^{j \tau} \frac{1}{1-v^{-1} v e^{r(t-y)}} e^{-\theta(y-\{j-1\} \tau)} d y\right\}+\int_{w \tau}^{t} \frac{1}{1-v^{-1} v e^{r(t-y)}} e^{-\theta(y-w \tau)} d y \\
= & \sum_{j=1}^{w}\left\{\int_{(j-1) \tau}^{j \tau} \sum_{k=0}^{\infty}\left(\frac{v}{v} e^{r t}\right)^{k} e^{-k r y-\theta(y-\{j-1\} \tau)} d y\right\}+\int_{w \tau}^{t} \sum_{k=0}^{\infty}\left(\frac{v}{v} e^{r t}\right)^{k} e^{-k r y-\theta(y-w \tau)} d y \\
= & \sum_{k=0}^{\infty} \frac{\left(\frac{v}{v} e^{r t}\right)^{k}}{k r+\theta}\left(\sum_{j=1}^{w}\left\{e^{-j k r \tau}\left(e^{k r \tau}-e^{-\theta \tau}\right)\right\}+e^{-k r w \tau}-e^{-\theta(t-w \tau)-k r t}\right) \\
= & \frac{1}{\theta}\left\{w\left(1-e^{-\theta \tau}\right)+1-e^{-\theta(t-w \tau)}\right\} \\
& +\sum_{k=1}^{\infty} \frac{\left(\frac{v}{v} e^{r t}\right)^{k}}{k r+\theta}\left(\frac{\left(1-e^{-k r w t}\right)\left(e^{-\theta \tau}-e^{k r \tau}\right)}{1-e^{k r \tau}}+e^{-k r w \tau}-e^{-\theta(t-w \tau)-k r t}\right),
\end{aligned}
$$

$\forall|v|<v$, if $r<0$, and $\forall|v|<v e^{-r t}$, if $r>0$. In fact the last expression in Eq. 35 holds for $w=0$ as well. Hence with Lemma 2.1 we obtain

$$
K_{Z_{t}}(v)=\frac{a_{0}}{r} \log \frac{v-v}{v e^{-r t}-v}+\frac{a_{1} \theta}{1-e^{-\theta \tau}} A(v, v)-\Lambda(t),
$$

for $r \neq 0$, and Eq. 34 follows after inserting $\Lambda(t)=a_{0} t+a_{1}\left\{\left(1-e^{-\theta(t-w \tau)}\right) /\right.$ $\left(1-e^{-\theta \tau}\right)+w$ \} into Eq. 36, which is due to Eqs. 3 and 33 .

\section{Saddlepoint Approximations to Tail Probabilities and Quantiles}

The saddlepoint approximation of asymptotic analysis provides accurate approximations to various complex integrals, like Fourier transforms. Daniels (1954) gives a saddlepoint approximation to the density of the mean of i.i.d. random variables and Lugannani and Rice (1980) give one to the distribution function. Result 3.1 below gives the Lugannani and Rice saddlepoint approximation to the tail probabilities of the inhomogeneous discounted compound Poisson process. Conditions for the existence of this saddlepoint approximation are given later under Lemma 3.1. These two last results are from Gatto (2010). We suggest a one-step inversion of this saddlepoint approximation, which yields an accurate approximation to the quantiles or values-at-risk. An upper quantile of the total claim amount is an essential index in the determination of the insurer capital. The one-step inversion is proposed by Wang (1995) for the sample mean and extended to M-estimators and conditional distributions by Gatto (2001). 
Result 3.1 Modified Lugannani and Rice saddlepoint approximation of the discounted total claim amount (Gatto 2010).

Let us define

$$
K(v)=\log \left\{e^{K_{Z_{t}}(v)}-e^{-\Lambda(t)}\right\}-\log \left\{1-e^{-\Lambda(t)}\right\},
$$

and let us denote $K^{\prime}(v)=(d / d v) K(v)$ and $K^{\prime \prime}(v)=(d / d v)^{2} K(v)$. Then the Lugannani and Rice saddlepoint approximation to $\bar{F}_{t}(x)=\mathrm{P}\left[Z_{t}>x\right]$ is given by

$$
\bar{G}_{t}(x)=\left[1-\Phi\left(r_{x}\right)+\phi\left(r_{x}\right)\left\{s_{x}^{-1}-r_{x}^{-1}\right\}\right]\left[1-e^{-\Lambda(t)}\right],
$$

where $s_{x}=v_{x}\left\{K^{\prime \prime}\left(v_{x}\right)\right\}^{1 / 2}, r_{x}=\operatorname{sgn}\left(v_{x}\right)\left\{2\left[v_{x} x-K\left(v_{x}\right)\right]\right\}^{1 / 2}, v_{x} \in \mathbb{R}$ is the saddlepoint implicitly defined as the solution in $v$ of

$$
K^{\prime}(v)=x
$$

and $x>0$ satisfies the conditions of Lemma 3.1 below.

Note that $K$ is the c.g.f. of the absolutely continuous random variable $Z_{t} \mid\left(N_{t}>0\right)$; i.e. $K=K_{Z_{t} \mid N_{t}>0}$. Lemma 3.1 below provides conditions under which the saddlepoint equation (Eq. 39) has an unique and real solution. The saddlepoint $v_{x}$ does not exist when $x$ belongs to a particular interval of $(0, \infty)$, which is called the "cemetery" of the saddlepoint.

Lemma 3.1 Existence of the saddlepoint approximation (Gatto 2010).

Suppose that $K_{Z_{t}}(v)$ exists for all $v \in(-\infty, \xi)$, for some $\xi \in[0, \infty]$.

(a) If $\xi=\infty$, then the cemetery of the saddlepoint is empty, precisely the empty set. This happens if $M_{X}(v)$ exists for all $v \in \mathbb{R}$.

(b) If $\xi<\infty$ and $K^{\prime}(\xi-)<\infty$, then the cemetery is the interval

$$
\left(x^{\dagger}, \infty\right) \stackrel{\text { def }}{=}\left(K^{\prime}(\xi-), \infty\right)=\left(\frac{K_{Z_{t}}^{\prime}(\xi-)}{1-e^{-\left\{K_{Z_{t}}(\xi-)+\Lambda(t)\right\}}}, \infty\right),
$$

where $K_{Z_{t}}^{\prime}(v)=(d / d v) K_{Z_{t}}(v)$ and $f(\xi-)=\lim _{v \rightarrow \xi, v<\xi} f(v)$, for a function $f$.

(c) If $\xi<\infty$, then the cemetery is empty iff

$$
K_{Z_{t}}^{\prime}(\xi-)=\infty
$$

A sufficient condition for Eq. 40 is that $K_{Z_{t}}$ is steep at $\xi$, i.e.

$$
K_{Z_{t}}(\xi-)=\infty \text {. }
$$

It can be deduced from Lemmas 2.1 and 3.1 that any bounded intensity with linear combination of exponentials claim amount distribution lead to an empty saddlepoint's cemetery. Indeed, we can see from Lemma 2.1 that the part of $K_{Z_{t}}(v)$ depending on $v$ is $\int_{0}^{t} M_{X}\left(v e^{r(t-y)}\right) \lambda(y) d y \geq 0$. Let $\lambda^{*}=\sup _{s \in[0, \tau)} \lambda(s)$. Then for $r \neq 0$ the previous integral is bounded from above by

$$
\lambda^{*} \int_{0}^{t} M_{X}\left(v e^{r(t-y)}\right) d y=\frac{\lambda^{*}}{r} \sum_{j=1}^{n} \mu_{j} \log \frac{v_{j}-v}{v_{j} e^{-r t}-v},
$$


$\forall v<\gamma_{t}=\min \left\{v_{1}, \ldots, v_{n}\right\} \min \left\{1, e^{-r t}\right\}$; see Eq. 15. This last expression tends to infinity as $v \rightarrow \xi=\gamma_{t}, v<\xi$, so the steepness condition in (c) of Lemma 3.1 guarantees that the saddlepoint's cemetery is empty.

Denote by $F_{t}^{*}$ and by $\bar{F}_{t}^{*}=1-F_{t}^{*}$ the distribution and the survivor functions of $Z_{t} \mid\left(N_{t}>0\right)$.

Remark 3.1 Chernoff bound.

Let $x>\mathrm{E}\left[Z_{t} \mid N_{t}>0\right]$ not in the saddlepoint's cemetery. Then the LegendreFenchel transform of $K$

$$
\delta_{t}(x) \stackrel{\text { def }}{=} \sup _{v<\xi_{t}} v x-K(v)=v_{x} x-\log \left\{e^{K_{Z_{t}}\left(v_{x}\right)}-e^{-\Lambda(t)}\right\}+\log \left\{1-e^{-\Lambda(t)}\right\}
$$

gives the Chernoff bound to the survivor function of $Z_{t} \mid\left(N_{t}>0\right)$,

$$
\bar{F}_{t}^{*}(x) \leq e^{-\delta_{t}(x)}=e^{-v_{x} x}\left(e^{K_{Z_{t}}\left(v_{x}\right)}-e^{-\Lambda(t)}\right)\left(1-e^{-\Lambda(t)}\right)^{-1} .
$$

We have thus the Chernoff bound to the survivor function of $Z_{t}$,

$$
\bar{F}_{t}(x) \leq e^{-v_{x} x}\left(e^{K_{Z_{t}}\left(v_{x}\right)}-e^{-\Lambda(t)}\right) .
$$

Result 3.2 below provides an accurate approximation to the quantiles of the discounted total claim amount. It derives from two Newton-Raphson iterations starting at the asymptotic normal approximation, as proposed by Wang (1995).

Result 3.2 One-step saddlepoint approximation to the quantiles of the discounted total claim amount.

Let $\varepsilon \in\left(e^{-\Lambda(t)}, 1\right)$ and define

$$
\begin{gathered}
\varepsilon^{*}=\frac{\varepsilon e^{\Lambda(t)}-1}{e^{\Lambda(t)}-1}, \\
q_{0}(\varepsilon)=K_{Z_{t}}^{\prime}(0)+\left\{K_{Z_{t}}^{\prime \prime}(0)\right\}^{\frac{1}{2}} \Phi^{(-1)}(\varepsilon) \\
=e^{r t}\left\{\mu_{X, 1} L_{t}(r)+\sqrt{\mu_{X, 2} L_{t}(2 r)} \Phi^{(-1)}(\varepsilon)\right\}
\end{gathered}
$$

and the initial value

$$
q_{1}^{*}\left(\varepsilon^{*}\right)=q_{0}(\varepsilon)+\frac{\left[\Phi^{(-1)}\left(\varepsilon^{*}\right)\right]^{2}-z_{q_{0}(\varepsilon)}^{2}}{2 v_{q_{0}(\varepsilon)}},
$$

where $\mu_{X, j}=\mathrm{E}\left[X_{1}^{j}\right], j=1,2, L_{t}(u)=\int_{0}^{t} e^{-u y} \lambda(y) d y$ and

$$
z_{x}=r_{x}+\frac{1}{r_{x}} \log \frac{s_{x}}{r_{x}}
$$

for $x>0$ not in the cemetery. Then

$$
q_{2}^{*}\left(\varepsilon^{*}\right)=q_{1}^{*}\left(\varepsilon^{*}\right)+\frac{\left[\Phi^{(-1)}\left(\varepsilon^{*}\right)\right]^{2}-z_{q_{1}^{*}\left(\varepsilon^{*}\right)}^{2}}{2 v_{q_{1}^{*}\left(\varepsilon^{*}\right)}},
$$

is the one-step saddlepoint approximation to the $\varepsilon$-quantile of $F_{t}$. 
Proof The asymptotic normal approximation provides the starting value for this onestep approximation. From the order statistics property of the Poisson process (see e.g. p. 536 in Gatto 2010), $Z_{t}$ is equivalent in distribution to a sum $S_{t}$ of $N_{t}$ i.i.d. random variables, independent of $N_{t}$ and with individual distribution depending on $t$. We note that $\lim _{t \rightarrow \infty} \mathrm{E}\left[e^{i v N_{t} / \Lambda(t)}\right]=e^{i v}$, which implies $N_{t} / \Lambda(t) \stackrel{\mathrm{P}}{\longrightarrow} 1$. With a periodic intensity we have $\lim _{t \rightarrow \infty} \Lambda(t)=\infty$ and thus, under the regularity conditions of the Central limit theorem of Lyapounov, for example, we can expect

$$
\frac{S_{t}-\mathrm{E}\left[S_{t}\right]}{\sqrt{\operatorname{var}\left(S_{t}\right)}} \stackrel{\mathrm{d}}{\longrightarrow} \mathcal{N}(0,1),
$$

as $\quad t \rightarrow \infty$. From $\mathrm{E}\left[S_{t}\right]=K_{Z_{t}}^{\prime}(0)=\mu_{X, 1} e^{r t} L_{t}(r) \quad$ and $\quad \operatorname{var}\left(S_{t}\right)=K_{Z_{t}}^{\prime \prime}(0)=$ $\mu_{X, 2} e^{2 r t} L_{t}(2 r)$, Eq. 42 is indeed a normal approximation to the $\varepsilon$-quantile of $F_{t}=1-\bar{F}_{t}$. We can easily see that the $\varepsilon$-quantile of $F_{t}$ is equal to the $\varepsilon^{*}$-quantile of $F_{t}^{*}$, where $\varepsilon^{*}$ is given by Eq. 41 , and we want to approximate this $\varepsilon^{*}$-quantile. The approximation to $\bar{F}_{t}^{*}(x)$ given by

$$
\bar{H}_{t}^{*}(x)=1-\Phi\left(z_{x}\right)
$$

is known having the same accuracy as the Lugannani and Rice saddlepoint approximation; see Lemma 2.1 of Jensen (1992). It has the advantage of allowing for a simple relationship between $\varepsilon^{*}$ and an approximate $\varepsilon^{*}$-quantile. The initial value (Eq. 43) and one-step approximation (Eq. 44) are derived from expansions of $z_{x}^{2}$ at $x=q_{0}(\varepsilon)$ and $x=q_{1}^{*}\left(\varepsilon^{*}\right)$, respectively, based on Eq. 45. Explanations can be found in Section 2.1 of Wang (1995).

\section{Implementations and Numerical Accuracies}

In this section we show the effectiveness of the proposed saddlepoint approximations to tail probabilities and quantiles. We illustrate their numerical accuracies by comparing them to Monte Carlo distributions based on $10^{6}$ generations of inhomogeneous compound Poisson processes. Devroye (1986) gives various ways of generating inhomogeneous Poisson processes, which are shortly summarized in the appendix of Gatto (2010). We use the inversion method based on a numerical evaluation of $\Lambda^{(-1)}(s)=\inf \{y \geq 0 \mid \Lambda(y) \geq s\}, s \geq 0$. This method is inefficient in terms of computing time, but our aim is only to provide some reliable results for comparisons. All our saddlepoint approximations are computed instantaneously. All computations are done with Matlab on a basic Apple Mac Mini.

Example 2.1, Continuation Trigonometric polynomial intensity and linear combination of exponentials claim amount.

For the model given under Example 2.1, we consider the following values of the parameters: $\tau=8, t=10, n=3, \mu_{1}=3, \mu_{2}=-3, \mu_{3}=1, v_{1}=1, v_{2}=2, v_{3}=$ $3, m=2, \alpha_{0}=7, \alpha_{1}=1, \alpha_{2}=2, \beta_{1}=2, \beta_{2}=0, r=0.1$. The nonnegativity of the intensity can be checked by Fejér's Theorem 2.1. If we take $u_{0}=v_{0}=1$, then $u_{2}=$ $v_{2}=1 / 2$ are consistent with Eq. 8 and $u_{1}=7 / 6, v_{1}=-5 / 6$ are consistent with Eq. 7 . By Eq. 6 , any $\alpha_{0} \geq 41 / 9 \simeq 4.5556$ leads to a nonnegative trigonometric polynomial and so $\alpha_{0}=7$ also. The given linear combination of exponential distributions is the 
distribution of $E_{1}+E_{2}+E_{3}$, where the summands are independent and $E_{j}$ has a density $j e^{-j x}, j=1,2,3$.

The numerical accuracy of the Lugannani and Rice saddlepoint approximation to the survivor function $\bar{F}_{t}(x)=\mathrm{P}\left[Z_{t}>x\right]$ is shown in Fig. 1. The highest or first graph of Fig. 1 shows the error $\bar{G}_{t}-\bar{F}_{t}$ of the Lugannani and Rice approximation $\bar{G}_{t}$ given by Eq. 38 in Result 3.1, versus $\bar{F}_{t}$. The exact survivor function $\bar{F}_{t}$ is actually computed by $10^{6}$ Monte Carlo generations of the total claim amount. The second graph of Fig. 1 shows the corresponding relative error computed by $\left(\bar{G}_{t}-\bar{F}_{t}\right) / \min \left\{\bar{F}_{t}, 1-\bar{F}_{t}\right\}$, which acts similarly over both left and right tails, versus $\bar{F}_{t}$. The third or lowest graph of Fig. 1 shows again the relative error but now versus the the domain of the distribution. All three graphs show that the Lugannani and Rice formula is unstable near to $\mathrm{E}\left[Z_{t}^{*}\right]$, where both $r_{x}^{-1}$ and $s_{x}^{-1}$ become large; otherwise it is very accurate. The peaks at the extremities are presumably due to the Monte Carlo approximation.
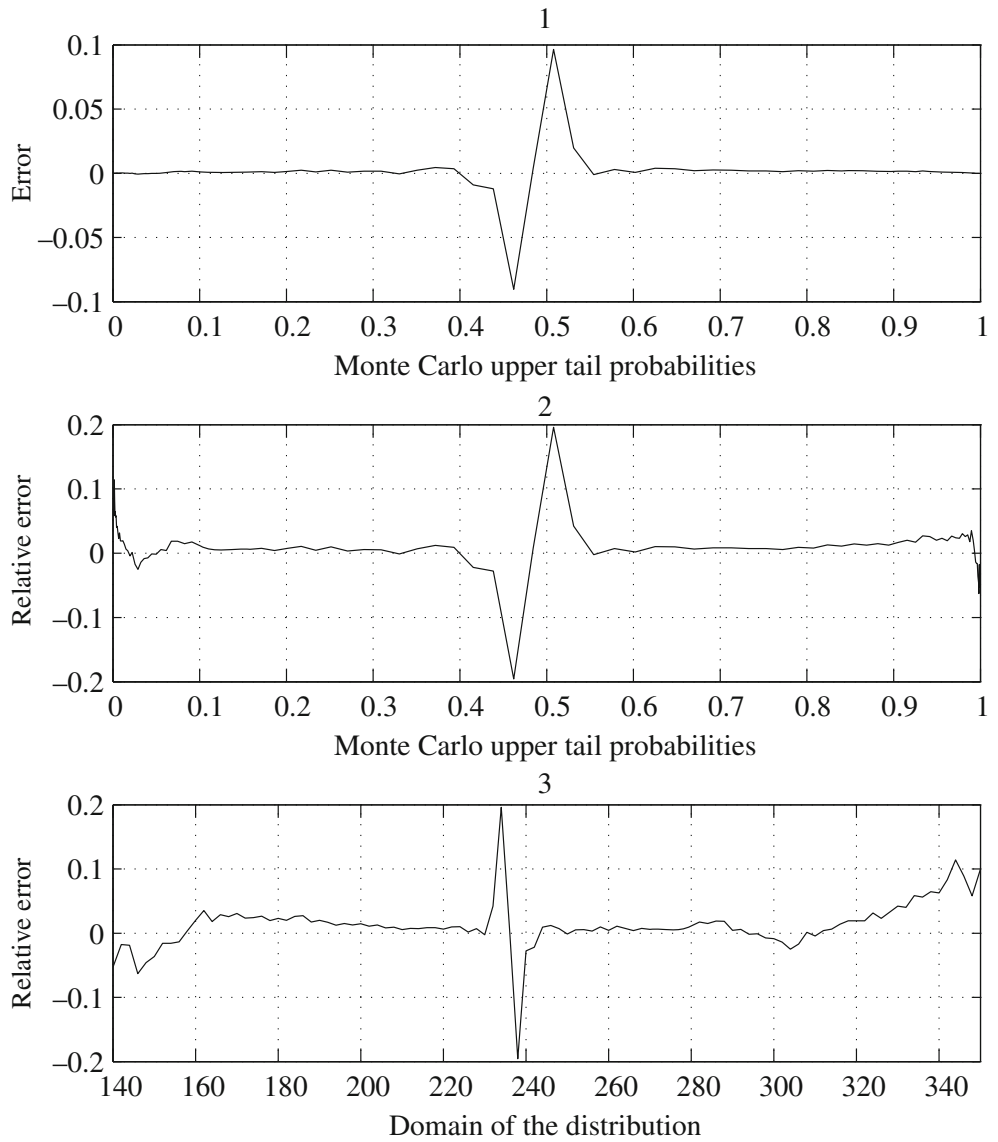

Fig. 1 Accuracy of the saddlepoint approximation to the upper tail probabilities $\bar{F}_{t}(x)=\mathrm{P}\left[Z_{t}>x\right]$ with trigonometric polynomial intensity and linear combination of exponentials claim amount. Graph 1: error, $\bar{G}_{t}-\bar{F}_{t}$ versus $\bar{F}_{t}$. Graph 2: relative error, $\left(\bar{G}_{t}-\bar{F}_{t}\right) / \min \left\{\bar{F}_{t}, 1-\bar{F}_{t}\right\}$ versus $\bar{F}_{t}$. Graph 3: relative error, $\left\{\bar{G}_{t}(x)-\bar{F}_{t}(x)\right\} / \min \left\{\bar{F}_{t}(x), 1-\bar{F}_{t}(x)\right\}$ versus $x$. $\bar{G}_{t}$ : Lugannani and Rice saddlepoint survivor 
Table 1 Accuracy of the one-step saddlepoint approximation to the quantiles of $F_{t}(x)=\mathrm{P}\left[Z_{t} \leq x\right]$ over the right tail with trigonometric polynomial intensity and linear combination of exponentials claim amount

\begin{tabular}{lllll}
\hline$\varepsilon$ & $q_{0}(\varepsilon)$ & $q_{1}^{*}\left(\varepsilon^{*}\right)$ & $q_{2}^{*}\left(\varepsilon^{*}\right)$ & $F_{t}\left(q_{2}^{*}\left(\varepsilon^{*}\right)\right)$ \\
\hline 0.800 & 264.706 & 264.388 & 264.298 & 0.8010 \\
0.850 & 271.345 & 271.393 & 271.350 & 0.8505 \\
0.900 & 279.696 & 280.386 & 280.395 & 0.9014 \\
0.950 & 292.071 & 294.037 & 293.970 & 0.9499 \\
0.960 & 295.675 & 298.089 & 298.006 & 0.9598 \\
0.970 & 300.106 & 303.079 & 302.973 & 0.9695 \\
0.980 & 305.995 & 309.781 & 309.637 & 0.9799 \\
0.990 & 315.276 & 320.483 & 320.287 & 0.9901 \\
0.999 & 341.278 & 351.503 & 351.048 & 0.9991 \\
\hline
\end{tabular}

$q_{0}(\varepsilon)$ asymptotic normal quantile. $q_{1}^{*}\left(\varepsilon^{*}\right)$ initial quantile. $q_{2}^{*}\left(\varepsilon^{*}\right)$ one-step quantile

Table 1 shows the performance of the one-step saddlepoint approximation to quantiles given under Result 3.2 for selected upper tail probabilities. The asymptotic normal quantile, the initial quantile and the one-step quantile, $q_{0}(\varepsilon) q_{1}^{*}\left(\varepsilon^{*}\right)$ and $q_{2}^{*}\left(\varepsilon^{*}\right)$, respectively, are given in Table 1 . The fifth column of Table 1 shows that the one-step saddlepoint quantiles are very accurate, because evaluating the exact or Monte Carlo distribution function at the one-step saddlepoint quantiles practically returns the initially selected upper tail probabilities.

Example 2.2, Continuation Wrapped $\alpha$-stable intensity and linear combination of exponentials claim amount.

For the model given under Example 2.2, we consider the following values of the parameters: $\tau=8, t=10, \alpha=1.4, \sigma=0.7, \beta=0.8, \mu=0, a_{0}=0, a_{1}=2, n=$ $3, \mu_{1}=3, \mu_{2}=-3, \mu_{3}=1, v_{1}=1, v_{2}=2, v_{3}=3, r=0.1$. By inserting these values into Eqs. 19 and 20, we obtain the cosine moments $\left(\gamma_{1}, \ldots \gamma_{9}\right)=(0.5764,0.0983$, $-0.0805,-0.0483,-0.0029,0.0045,0.0008,-0.0003,-0.0001)$ and sine mo-

Fig. $2 \mathrm{~W} \alpha \mathrm{S}$ intensity

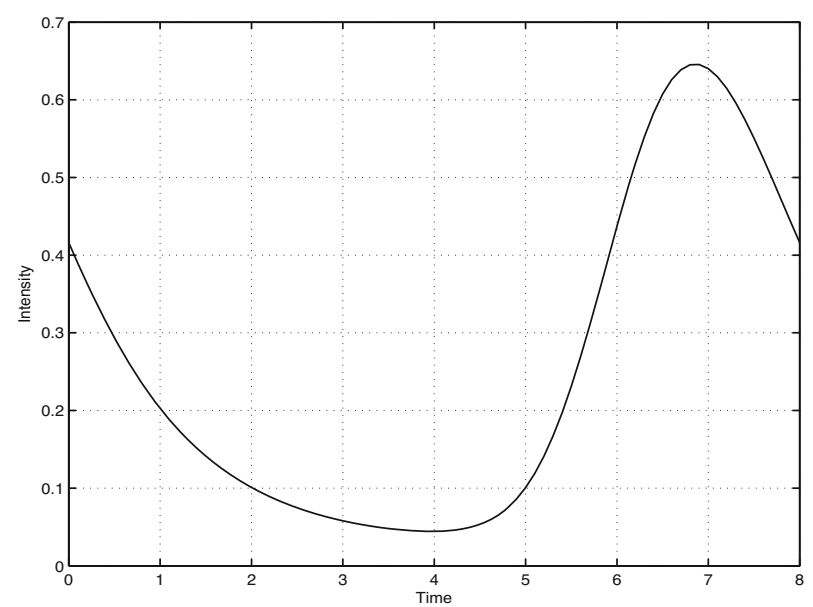


ments $\left(\delta_{1}, \ldots, \delta_{9}\right)=(-0.2976,-0.3036,-0.1063,0.0087,0.0160,0.0020,-0.0011$, $-0.0002,0.0001)$. Higher cosine and sine moments are close to zero and thus neglected. We can thus use the expansion to the c.g.f. of Result 2.1 where the infinite sum is actually limited to the first nine summands, which means that we are using Eq. 12 with $m=9$. The $\mathrm{W} \alpha \mathrm{S}$ intensity is shown in Fig. 2 over one period and we can see that it is circularly right-skewed.

Table 2 shows the accuracy of the saddlepoint approximation to the exact survivor function $\bar{F}_{t}(x)=\mathrm{P}\left[Z_{t}>x\right]$, which is again based on $10^{6}$ Monte Carlo simulations, with the above $\mathrm{W} \alpha \mathrm{S}$ intensity and linear combination of exponential claim amounts. $\bar{G}_{t}$ denotes the Lugannani and Rice saddlepoint approximation and $\bar{H}_{t}$ the asymptotic equivalent formula (Eq. 45). The relative errors, defined as under the continuation of Example 2.1 above, are shown in the fifth and sixth columns. They are very small and uniformly bounded. The central part of the distribution

Table 2 Accuracy of the saddlepoint approximation to the survivor function $\bar{F}_{t}(x)=\mathrm{P}\left[Z_{t}>x\right]$ over the left and right tails with $\mathrm{W} \alpha \mathrm{S}$ intensity and linear combination of exponential claim amounts

\begin{tabular}{|c|c|c|c|c|c|}
\hline$x$ & $\bar{F}_{t}(x)$ & $\bar{G}_{t}(x)$ & $\bar{H}_{t}(x)$ & $\frac{\bar{G}_{t}(x)-\bar{F}_{t}(x)}{\min \left\{\bar{F}_{t}(x), 1-\bar{F}_{t}(x)\right\}}$ & $\frac{\bar{H}_{t}(x)-\bar{F}_{t}(x)}{\min \left\{\bar{F}_{t}(x), 1-\bar{F}_{t}(x)\right\}}$ \\
\hline 2.9 & 0.7494 & 0.7375 & 0.7387 & -0.0472 & -0.0424 \\
\hline 3.1 & 0.7336 & 0.7200 & 0.7213 & -0.0509 & -0.0461 \\
\hline 3.3 & 0.7166 & 0.7021 & 0.7034 & -0.0512 & -0.0463 \\
\hline 3.5 & 0.7000 & 0.6838 & 0.6853 & -0.0537 & -0.0488 \\
\hline 3.7 & 0.6840 & 0.6652 & 0.6668 & -0.0594 & -0.0544 \\
\hline 3.9 & 0.6675 & 0.6463 & 0.6480 & -0.0639 & -0.0588 \\
\hline 4.1 & 0.6523 & 0.6270 & 0.6288 & -0.0730 & -0.0677 \\
\hline 4.3 & 0.6365 & 0.6070 & 0.6091 & -0.0809 & -0.0754 \\
\hline 4.5 & 0.6202 & 0.5868 & 0.5890 & -0.0880 & -0.0822 \\
\hline 4.7 & 0.6038 & 0.5656 & 0.5681 & -0.0965 & -0.0903 \\
\hline 4.9 & 0.5879 & 0.5436 & 0.5464 & -0.1076 & -0.1008 \\
\hline 11.0 & 0.2150 & 0.1984 & 0.1992 & -0.0771 & -0.0733 \\
\hline 12.0 & 0.1768 & 0.1699 & 0.1704 & -0.0388 & -0.0361 \\
\hline 13.0 & 0.1446 & 0.1419 & 0.1422 & -0.0185 & -0.0162 \\
\hline 14.0 & 0.1174 & 0.1168 & 0.1170 & -0.0054 & -0.0033 \\
\hline 15.0 & 0.0957 & 0.0953 & 0.0955 & -0.0043 & -0.0023 \\
\hline 16.0 & 0.0781 & 0.0771 & 0.0773 & -0.0130 & -0.0111 \\
\hline 17.0 & 0.0628 & 0.0621 & 0.0623 & -0.0099 & -0.0080 \\
\hline 18.0 & 0.0502 & 0.0498 & 0.0499 & -0.0090 & -0.0071 \\
\hline 19.0 & 0.0398 & 0.0397 & 0.0397 & -0.0035 & -0.0016 \\
\hline 20.0 & 0.0312 & 0.0315 & 0.0316 & 0.0097 & 0.0116 \\
\hline 21.0 & 0.0245 & 0.0249 & 0.0250 & 0.0164 & 0.0184 \\
\hline 22.0 & 0.0191 & 0.0197 & 0.0197 & 0.0325 & 0.0345 \\
\hline 23.0 & 0.0150 & 0.0155 & 0.0155 & 0.0322 & 0.0342 \\
\hline 24.0 & 0.0114 & 0.0121 & 0.0121 & 0.0608 & 0.0629 \\
\hline 25.0 & 0.0089 & 0.0095 & 0.0095 & 0.0667 & 0.0688 \\
\hline 26.0 & 0.0068 & 0.0074 & 0.0074 & 0.0881 & 0.0902 \\
\hline 27.0 & 0.0053 & 0.0057 & 0.0057 & 0.0880 & 0.0902 \\
\hline 28.0 & 0.0040 & 0.0044 & 0.0045 & 0.1196 & 0.1218 \\
\hline 29.0 & 0.0031 & 0.0034 & 0.0034 & 0.0950 & 0.0972 \\
\hline 30.0 & 0.0024 & 0.0027 & 0.0027 & 0.0972 & 0.0993 \\
\hline
\end{tabular}

Relative errors are shown in the fifth and sixth columns. $\bar{G}_{t}$ : Lugannani and Rice saddlepoint survivor. $\bar{H}_{t}$ : equivalent saddlepoint survivor 
is not shown in Table 2, again because of the instability of the Lugannani and Rice saddlepoint approximation and its equivalent formula (Eq. 45) near to the center, i.e. for small saddlepoint values. The lack of stability at the center is a minor drawback, because upper tail probabilities or values-at-risk are the most useful in many actuarial problems, for example in the constitution of reserves. For other problems where central probabilities or values-at-risk are required, one could first compute the saddlepoint approximation to the density by Daniels (1954) and then integrate it numerically. The values on the left tail cannot be obtained beyond $x=2.9$ because the saddlepoints $v_{x}$ go below $\gamma_{t}=1 / e$ for $x$ below 2.9, approximately, where the expansion to $K_{Z_{t}}$ (Eq. 18) does not hold anymore. As Eq. 18 holds over $\left(-\gamma_{t}, \gamma_{t}\right)$, whereas Lemma 3.1 assumes that $K_{Z_{t}}$ exists over $\left(-\infty, \gamma_{t}\right)$, this problem over the left tail is not identified by Lemma 3.1. The saddlepoint approximation could be computed over the left tail by integrating numerically the c.g.f. as given under Lemma 2.1. Note also that we have $\Lambda(t)=2.4807$ and therefore $1-\exp \{-\Lambda(t)\}=0.9163$ is the total probability beyond the jump at $x=0$.

\section{Final Remarks}

In the first part of this article we suggest various types of periodic intensity functions for the insurer compound Poisson total claim amount subject to a fixed force of interest. We also provide series representations for the c.g.f. of the total claim amount distributions, under the proposed periodic intensities. Next, we suggest inverting these c.g.f. by the saddlepoint approximation, in order to obtain fast and accurate approximations to tail probabilities. We then provide an algorithm for computing the related quantiles or values-at-risk. The accuracy of the methods proposed is illustrated by two numerical examples. In these examples, the saddlepoint approximation is numerically very close to Monte Carlo simulation. The saddlepoint approximation seems also promising for computing stabilities of tail probabilities; that is, variations w.r.t. variations of model parameters. Saddlepoint approximations for other models of total claim amount would also be useful. For example, Gatto (2011) considers the doubly compound total claim amount, where a primary counting birth process is used for the number of catastrophic events and a secondary counting distribution is used for the number of individual claims generated from each catastrophe of the primary process.

Acknowledgements The author thanks the editor and a referee for substantive suggestions which improved the quality of this article.

\section{References}

Abramowitz M, Stegun IE (1972) Handbook of mathematical functions with formulas, graphs, and mathematical tables. 9th printing by Dover. Originally published by the National Bureau of Standards, USA. 10th printing

Albrecher H, Asmussen S (2006) Ruin probabilities and aggregate claims distributions for shot noise Cox processes. Scand Actuar J 2:86-110

Batschelet E (1981) Circular statistics in biology. Academic, New York

Daniels HE (1954) Saddlepoint approximations in statistics. Ann Math Stat 25:631-650

Devroye L (1986) Non-uniform random variate generation. Springer, Berlin 
Dimitrov DK (2002) Extremal positive trigonometric polynomials. In: Approximation theory: a volume dedicated to Blagovest Sendov. Darba, Sofia, pp 136-157

Fejér L (1915) Uber trigonometrische Polynome. J Reine Angew Math 146:53-82

Gatto R (2001) Symbolic computation for approximating the distributions of some families of one and two-sample nonparametric test statistics. Stat Comput 11:449-455

Gatto R (2008) Some computational aspects of the generalized von Mises distribution. Stat Comput 18:321-331

Gatto R (2010) A saddlepoint approximation to the distribution of inhomogeneous discounted compound Poisson processes. Methodol Comput Appl Probab 12:533-551

Gatto R (2011) Values- and tail-values-at-risk of doubly compound inhomogeneous and contagious aggregate loss processes. Technical report, Institute of Mathematical Statistics and Actuarial Science, University of Bern, Switzerland

Gatto R, Jammalamadaka SR (2003) Inference for wrapped symmetric $\alpha$-stable circular models. Sankhyā 65:333-355

Gatto R, Jammalamadaka SR (2007) The generalized von Mises distribution. Stat Methodol 4:341-353

Jammalamadaka SR, SenGupta A (2001) Topics in circular statistics. World Scientific, Singapore

Jensen JL (1992) The modified signed likelihood statistic and saddlepoint approximations. Biometrika 79:693-703

Léveillé G, Garrido J, Wang YF (2010) Moment generating functions of compound renewal sums with discounted claims. Scand Actuar J 3:165-184

Lugannani R, Rice S (1980) Saddle point approximation for the distribution of the sum of independent random variables. Adv Appl Probab 12:475-490

Mardia KV, Jupp PE (2000) Directional statistics. Wiley, New York

Pinkus A, Zafrany S (1997) Fourier series and integral transforms. Cambridge University Press, Cambridge

Rudin W (1987) Real and complex analysis. McGraw-Hill, New York

Samorodnitsky G, Taqqu MS (1994) Stable non-Gaussian random processes: stochastic models with infinite variance. Chapman \& Hall, London

Wang S (1995) One-step saddlepoint approximations for quantiles. Comput Stat Data Anal 20:65-74 